\title{
Wavelet Based Video Denoising using Probabilistic Models
}

\author{
AZKA MAQSOOD*, IMRAN TOUQIR*, ADIL MASOOD SIDDIQUI*, AND MAHAM HAIDER* \\ RECEIVED ON 26.09.2017 ACCEPTED ON 13.11.2017
}

\begin{abstract}
Wavelet based image processing techniques do not strictly follow the conventional probabilistic models that are unrealistic for real world images. However, the key features of joint probability distributions of wavelet coefficients are well captured by HMT (Hidden Markov Tree) model. This paper presents the HMT model based technique consisting of Wavelet based Multiresolution analysis to enhance the results in image processing applications such as compression, classification and denoising. The proposed technique is applied to colored video sequences by implementing the algorithm on each video frame independently. A 2D (Two Dimensional) DWT (Discrete Wavelet Transform) is used which is implemented on popular HMT model used in the framework of Expectation-Maximization algorithm. The proposed technique can properly exploit the temporal dependencies of wavelet coefficients and their non-Gaussian performance as opposed to existing wavelet based denoising techniques which consider the wavelet coefficients to be jointly Gaussian or independent. Denoised frames are obtained by processing the wavelet coefficients inversely. Comparison of proposed method with the existing techniques based on CPSNR (Coloured Peak Signal to Noise Ratio), PCC (Pearson's Correlation Coefficient) and MSSIM (Mean Structural Similarity Index) has been carried out in detail. The proposed denoising method reveals improved results in terms of quantitative and qualitative analysis for both additive and multiplicative noise and retains nearly all the structural contents of a video frame.
\end{abstract}

Key Words: Video Denoising, Wavelet Multiresolution Analysis, Additive and Multiplicative Noise, Hidden Markov Tree Model, Expectation Maximization.

\section{INTRODUCTION}

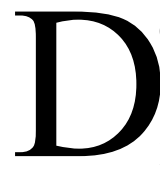
enoising can effectively enhance visual quality and considerably simplify the subsequent processing tasks like video compression and pattern recognition. Video denoising considers spatiotemporal data of video signal and it is different from image denoising. It can be achieved by different approaches: spatial-domain, temporal-domain, and spatial-temporal combination. Different techniques have exploited the correlation between the reference frames (i.e. the past and the future frame). Spatial domain methods of denoising are limited in their scope as these methods do not take temporal correlation between frames into account [1-3]. Wiener filter is an example of a spatial filter that removes spatial noise from images and succeeds in achieving high gain. However, this filter cannot restore edges especially in less noisy areas [4].

Authors E-Mail: (azka.msee21@students.mcs.edu.pk, imrantqr@mcs.edu.pk, dradil@mcs.edu.pk, mahamhaider.msee19@students.mcs.edu.pk) * Military College of Signals, National University of Science \& Technology, Rawalpindi, Pakistan.

This is an open access article published by Mehran University Research Journal of Engineering and Technology, Jamshoro under the CC by 4.0 International License. 
Temporal domain methods consider the inter-frame correlation between frames and perform well for still videos without motion [5]. In the case of videos having motion, temporal domain methods do not provide significant results. Ozkan et. al. [6] proposed a temporal filter for denoising of frames that provide considerably good results in the noise removal process and produce less blocking artifacts but it causes blurring effect.

Liu and Luo [7] introduced a method based on TV (Total Variation) and temporal filtering for image denoising. The temporal filter maintains structure and edges well but it cannot reduce noise. The TV algorithm is applied to a noisy frame to reduce noise but it could not restore structure information.

Spatio-temporal methods consider both spatial and temporal correlations between different frames in a video sequence and provide efficient results.

Maggioni et. al. [8] have addressed the problem of denoising in video sequences that are corrupted by random and fixed pattern noise. In this method, the data is sparsified in 3D spatiotemporal transform domain by leveraging the spatial and temporal correlations within each volume. 3D threshold array is used to shrink the coefficients of the 3D volume spectrum.

Wang et. al. [9] proposed a spatial-temporal depth filter by jointly using the depth and texture information in the spatial-temporal domain that performs filtering in three steps. First, a pixel to be filtered and its reference pixels are selected based on the similar pixel vectors. Second, the most correlated pixels are recognized among reference pixels. Finally, the to-be-filtered pixel is obtained by using a median filter among the reference pixels.
Transform domain methods exploit the sparsity of data and have good localization properties and multiresolution characteristics in either temporal domain or frequency domain. These properties make it more useful to separate a useful signal from noise. Hence, wavelet has gained popularity for image denoising [10-11]. Wavelet transform can be $2 \mathrm{D}$ or 3D. 3D transform domain methods do not perform well for denoising purpose because of long delay and inability to adapt to fast motions in a video sequence [12].

Zhigang et. al. [13] proposed wavelet based threshold function to overcome the discontinuity of the hard threshold method and the soft threshold method at the threshold value.

Ho and Hwang [15] proposed an image noise reduction method through the wavelet domain Bayesian threshold criterion coefficient of shrinkage method.

Techniques like VBM3D and E-RF3D have been the most efficient ones in denoising as they exploit DCT (Discrete Cosine Transform) in their framework [16].

Non-local means technique proposed in [17] performing efficient denoising and have become popular in recent years. Some of the denoising methods are based on motion estimation and compensation process as the removal of noise and visual quality is mainly dependent on the amount of motion occured in a video sequence.

Zuo et. al. [18] proposed a video noise removal method to exploit spatial-temporal correlations between different frames. First of all, the motion is estimated between current noisy and previously denoised frames and then Kalmanbilateral filtering is applied to the current noisy frame.

Aydin and Foroosh [19] proposed wavelet based METF (Motion Estimation Temporal Filtering) that applies ME directly on wavelet coefficients. 
Hong-Zhi et. al. [20] introduced a spatiotemporal method to minimize noise in video frames by discriminating the still regions from moving regions. In this technique, Kalman bilateral filtering is applied to still regions that do not show any motion and spatial bilateral filtering is performed on moving regions.

Although there are a number of denoising techniques but there is always a room for improvement. The proposed method is an extension of HMT based framework used in $[14,21]$.

In [14], HMT based statistical signal processing technique is proposed for compression of signals in wavelet domain. Whereas in [21], denoising of gray scale images using HMT is presented. Using the above modeling framework, a combined spatial and temporal filtering technique is proposed in this paper that can remove Gaussian as well as speckle noise from color image and video sequences considerably. By exploiting the dependencies among wavelet coefficients, better performance has been achieved. The proposed method deals with non-Gaussian behavior of wavelet coefficients that are often encountered in practice and gives efficient results for de-

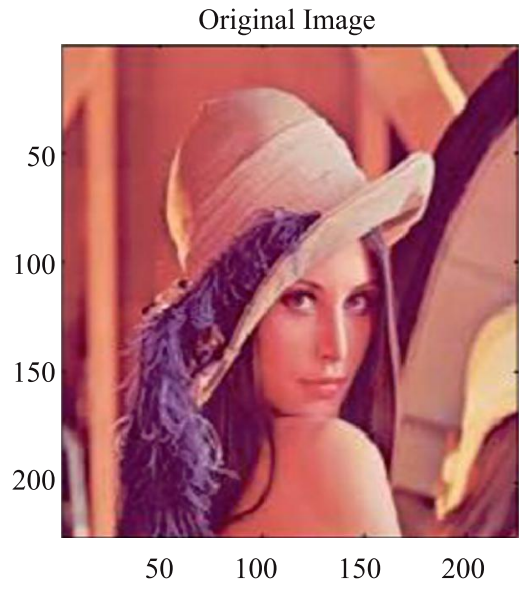

(a) Original Image speckling of images as well. The results show that the proposed method dose not remove noise only but also retains almost all the structural information of a video frame.

The remaining part of this paper is organized as follows. In Section 2, we discuss about the wavelet-based denoising model. In Section 3, modeling of wavelet coefficients by using HMT model is elaborated. Section 4 defines the proposed denoising model. In Section 5 the experimental results and discussion of the proposed model are given. Finally, we conclude this paper in the last section.

\section{WAVELET-BASED VIDEO DENOISING}

A WT leads to a sparse and efficient representation of an image as it hybrid the spatial and transform domain. 2DDWT has been used in this paper.

DWT decompose the image into one low-frequency subband and several high-frequency sub-bands in such a way that most of the important information is concentrated in LL sub-band of the highest level also known as DWT approximation as shown in Fig. 1(a-b).

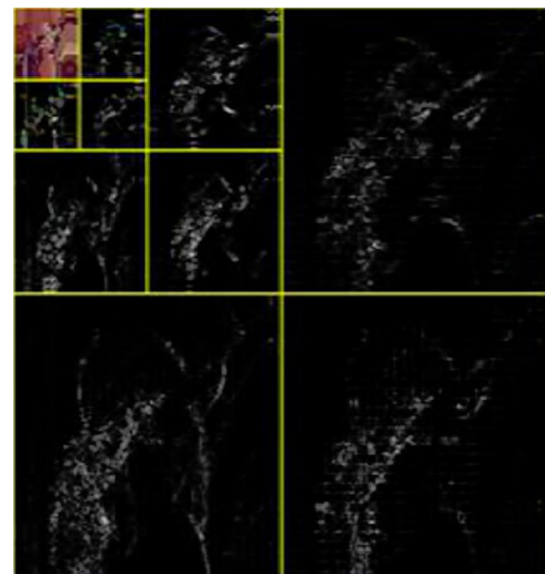

(b) DWT Decomposition

FIG. 1. THREE LEVEL DWT DECOMPOSITION OF LENA IMAGE

Mehran University Research Journal of Engineering \& Technology, Volume 38, No. 1, January, 2019 [p-ISSN: 0254-7821, e-ISSN: 2413-7219] 


\section{MODELLING FOR VIDEO DENOISING USING HMM}

HMM (Hidden Markov Model) captures the nonGaussian statistics and complex dependencies among wavelet coefficients referred to as clustering property and persistence property respectively.

The HMM uses a quad-tree structure and has also been successfully used in the Bandelet domain and contourlet domain. An m-state HMM links each wavelet coefficient with a hidden state variable in such a way that each wavelet coefficient is characterized by an m-dimensional state probabilities vector $\mathrm{q}$ and an $\mathrm{m}$-dimensional standard deviation vector $\sigma$.

$\mathrm{q}=\left(\mathrm{q}_{1}, \mathrm{q}_{2}, \ldots, \mathrm{q}_{\mathrm{m}}\right)^{\mathrm{t}}$
$\sigma=\left(\mathrm{s}_{1}, \mathrm{~s}_{2}, \ldots, \mathrm{s}_{\mathrm{m}}\right)^{\mathrm{t}}$

\subsection{Capturing Non-Gaussian Densities}

The non-Gaussian density of wavelet coefficients can be captured efficiently by GMM (Gaussian Mixture Model) and a multidimensional GMM is referred to as HMT. HMT models the wavelet coefficients as random variables having probability density function as a mixture of zero mean Gaussian distributions by means of a hidden state to designate small and large coefficient.

The pdf of the wavelet coefficient $C$ is defined as:

$f_{c}(c)=\sum_{n=1}^{N} p_{s}(n) f_{c \mid S}(c \mid S=n)$

where $\mathrm{p}_{\mathrm{s}}(\mathrm{n})$ is pmf(probability mass function), $\mathrm{S}$ is the hidden state variable which is invisible and it controls the magnitude of wavelet coefficient.
The $f_{C \mid S}(c \mid S=n)$ is the conditional pmf given by the following Equation (4):

$f_{c \mid S}(c \mid S=n)=\frac{1}{\sigma_{n} \sqrt{2 \pi}} \exp \left(-\frac{\left(b-\mu_{n}\right)^{2}}{2 \sigma_{n}^{2}}\right)$

where $\mu_{\mathrm{n}}$ and $\sigma_{\mathrm{n}}$ are the mean and variance respectively.

\subsection{Capturing Dependencies}

For capturing the interscale and intrascale dependencies among wavelet coefficients, HMT uses the probabilistic tree to model the Markovian dependencies between hidden states. To a wavelet decomposition of $J$ scale and $K$ subband, an HMT model contains the following parameters:

$\mathrm{P}_{\mathrm{si}}(\mathrm{n})$ : pmf for the node root node $\mathrm{S}_{\mathrm{i}}$

$\mathrm{A}_{\mathrm{j}, \mathrm{k}}$ a state transition probability matrix of $k$ sub-band from scale $j-1$ to scale $j$

$\mu_{\mathrm{jk},}, \sigma_{\mathrm{j}, \mathrm{k}}:$ Gaussian mean and standard deviation vector of wavelet coefficient in $j$ scale and $k$ sub-band.

The state transition matrix shows parent $\rightarrow$ children state to state links between the hidden states that is given as:

$A_{j, k}=\left[\begin{array}{cc}p_{j, k}^{u \rightarrow u} & p_{j, k}^{u \rightarrow v} \\ p_{j, k}^{v \rightarrow u} & p_{j, k}^{v \rightarrow v}\end{array}\right]$

where $p_{j, k}^{u \rightarrow w}$ shows the child coefficient is in state $u$ given parent coefficient is in state $w$ [23]. Also, $p_{j, k}^{u \rightarrow u}$ or $p_{j, k}^{v \rightarrow v}$ represent the probability of a wavelet coefficient to be small or large given its parent is small or large. All the parameters of the HMT model are grouped together in the form of vector $\theta$ given in Equation (6).

$\theta=\left\lfloor p\left(S_{i}=n\right), A_{j, k}, \mu_{j, k}, \sigma_{j, k}\right\rfloor$ 
It is to be noted here that each wavelet coefficient has different variances and state transition probabilities which lead to greater complexity in the HMT model. We can reduce this computational complexity by a method of tying within scale [11]. According to this method, the wavelet coefficients have the same density within a scale.

\section{DENOISING TECHNIQUE}

HMT based denoising technique in the perspective of 2D GMM (Gaussian Mixture Models) and 2D DWT is used by applying it to each video frame independently. EM (Expectation-Maximization) algorithm iteratively finds the maximum likelihood of a fundamental distribution from a given data set. Our proposed method exploits the effectiveness of DWT and the hierarchical relationships between its sub-bands.

\subsection{Noisy wavelet Coefficients}

Let $\mathrm{Q}$ be a natural clean image with $\mathrm{NxN}$ dimension and Q' be its noisy version such that $\mathrm{Q}^{\prime}=\mathrm{E}$ where $\mathrm{E}$ is zero mean white Gaussian noise. By performing wavelet decomposition on Q' the wavelet coefficient q'is obtained. Due to the linearity of the wavelet transform, we have:

$q^{\prime}=q+e$

where $\mathrm{q}$ and $\mathrm{e}$ are the wavelet coefficients of $\mathrm{Q}$ and $\mathrm{E}$ respectively. We need to estimate the q given q'.

\subsection{Model Parameters Determination}

HMT model is used to find a set of parameters $\theta_{\mathrm{q}}$. Initially, a two-state GMM is used to characterize each wavelet coefficient and a noisy observation is used to initiate the HMT model. Then the interscale dependencies are captured by the Markov-tree and EM algorithm is used to obtain $\theta_{q}$.
According to [10], the added noise in a signal only increases its variance by leaving the other parameters unchanged. Hence, the noise free observation $\theta_{\mathrm{q}}$ can be extracted by fitting the HMT to the noisy observation and then subtracting the noise variance from it.

$$
\left(\sigma_{(j, k, m), n}^{(q)}\right)^{2}=\left(\left(\sigma_{(j, k, m), n}^{\left(q^{\prime}\right)}\right)^{2}-\left(\sigma_{(j, k, m)}^{(e)}\right)^{2}\right)_{+}
$$

where j,k,m represent $j$ scale, $k$ sub-band and $n$ state, $m$-th coefficient and $(\mathrm{g})_{+}=\mathrm{g}$ for $\mathrm{g} \geq 0$ and $(\mathrm{g})_{+}=0$ for $\mathrm{g}<0$. Noise variance $\left(\sigma_{(j, k, m), n}^{(q)}\right)^{2}$ can be estimated by median estimator in finest sub-band [22].

\subsection{Clean Coefficients}

To determine the noise free coefficients, the EM algorithm is used for training the model. There is a need to determine the noise free coefficients $q$ from q'. Generalizing it, this model refers to determining the vector denoted by $\theta_{\mathrm{q}}$. The following relation of expectation describes the sufficient statisticsof the model for variable $\mathrm{C}$ and the hidden state variable $\mathrm{S}$ :

$E_{\theta_{o}}\left(S_{t}(C, S) C=c\right)=E_{\theta} S_{t}(C, S)$

The conditional pmf of hidden states $\mathrm{S}$ and its maximization is given by following expressions:

$$
\begin{aligned}
& P\left(S=n \mid c, \theta_{q}\right)=\frac{P\left(S_{i}=k\right) g\left(c ; 0, \sigma_{j, k}^{2}\right)}{\sum_{i=0}^{1} P\left(S_{i}=l\right) g\left(c ; 0, \sigma_{j, l}^{2}\right)} \\
& P(S=n)=\frac{1}{N_{i}} \sum_{b \in Z^{2}} P\left(S=n \mid c, \theta_{q}\right)
\end{aligned}
$$

Once $\theta_{\mathrm{q}}$ is determined [10] and state probability is given through HMT, we can get $E=\left\lfloor q \mid q^{\prime}, \theta_{q}\right\rfloor$ by using Bayes estimator to get the clean coefficients: 


$$
q=E\left[q \mid q^{\prime}, \theta_{q}\right]=\sum_{n}\left(S \mid q, \theta_{q}\right) \times \frac{\left(\sigma_{(j, k, m), n}^{(q)}\right)^{2}}{\left(\sigma_{(j, k, m), n}^{\left(q^{\prime}\right)}\right)^{2}+\left(\sigma_{(j, k, m)}^{(e)}\right)^{2}} q_{j, k, m}^{\prime}
$$

where $\mathrm{j}, \mathrm{k}$, denote the $m$-th coefficient in scale $j$ and subband $k$.

\subsection{Reconstructed Frames}

At the end, the inverse wavelet transform is applied to the obtained clean coefficients to get the reconstructed frames of a video sequence.

Fig. 2 shows step wise implementation of the proposed technique.

The algorithm for the proposed denoising technique can be summarized as follows:

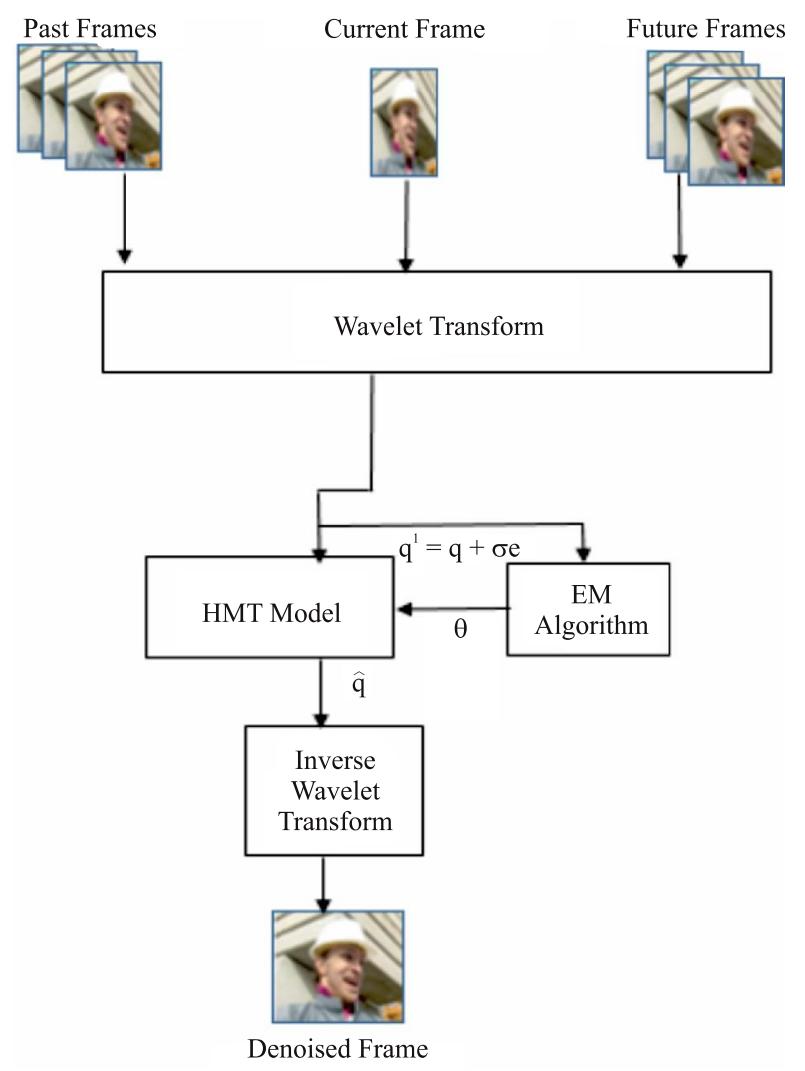

FIG. 2. DIAGRAM OF PROPOSED DENOISING PROCESS

\section{Denoising Algorithm}

Add AWGN noise to the video sequence

Apply Daubechies-8 DWT on each frame

Obtain DWT coefficients

Calculate the GMM parameters of each sub-band.

Train the HMT model using EM algorithm with reference to tying within scale method.

Apply inverse wavelet transform to get the reconstructed frames.

\section{SIMULATIONAND EXPERIMENTS}

To illustrate the efficiency of the proposed algorithm, different standards publicly available test video sequences are used such as BUS, MOBILE, SALESMAN, CHAIR, FOOTBALL andFOREMAN. Each sequence is artificially degraded with white Gaussian noise and speckle noise. The reconstructed frame is tested with the original one. Qualitative analysis is performed in Figs. 3-5 with existing state-of-the-art methods including NLM, VBM3D and CIFIC [17,18,24]. The block in original frame show zoomed regions and its comparison with other techniques.

NLMC, CIFIC and V-BM3D do not consider speckle noise while proposed method outperforms the existing despeckling techniques. In Figs. 6-9, the original frame corrupted with speckle noise is filtered by using different techniques including SOMA, MD, Wavelets [24-26] and proposed algorithm.

The quantitative results are shown in Tables 1-4. Tables 1-2 show better performance of the proposed algorithm in terms ofCPSNR, MSSIM and PCC.

CIFIC considers both uniform and non-uniform Gaussian noise while V-BM3D and NLMC onlyperform well for uniform Gaussian noise. Hence, the comparison is made here only for the case of uniform noise in video sequences.

Mehran University Research Journal of Engineering \& Technology, Volume 38, No. 1, January, 2019 [p-ISSN: 0254-7821, e-ISSN: 2413-7219] 


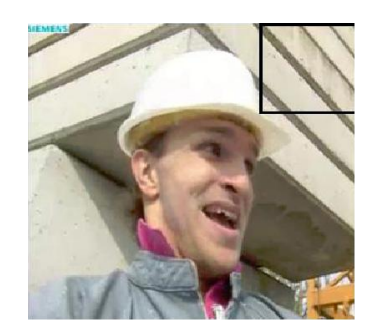

FIG. 3(a). ORIGINAL IMAGE
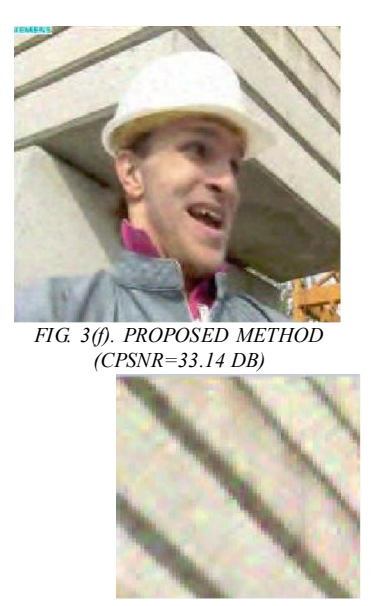

FIG. 3(j). SAME ZOOMED AREA OF 'D'

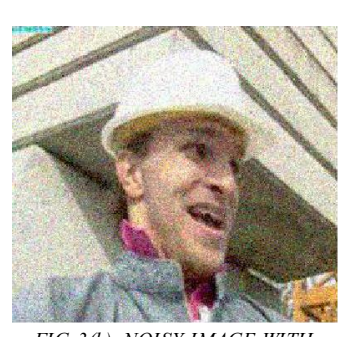

FIG. 3(b). NOISY IMAGE WITH

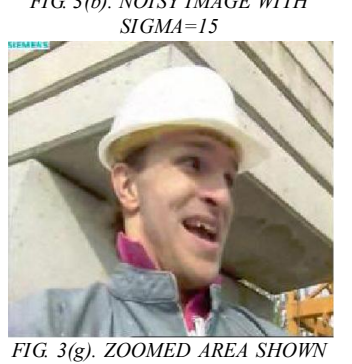

BYA BLOCK IN 'A'
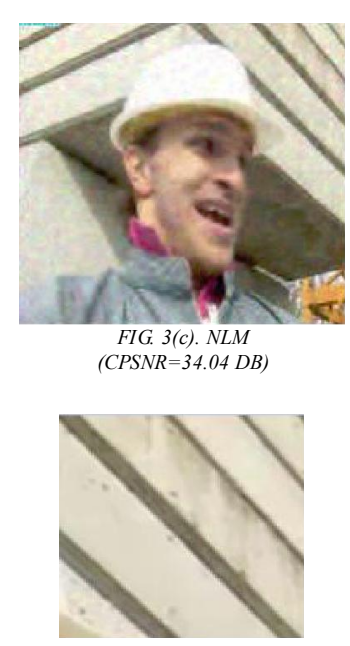

FIG. 3(h). SAME ZOOMED AREA $O F{ }^{\prime} B^{\prime}$
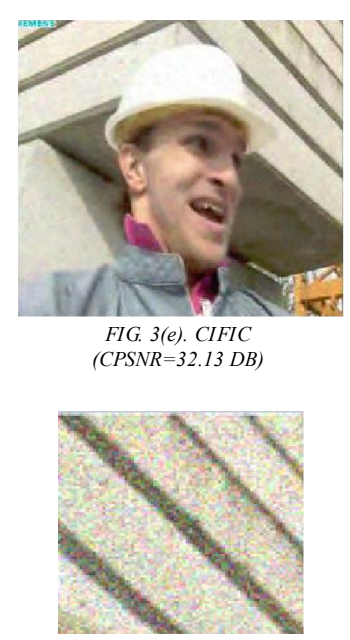

FIG. 3(i). SAME ZOOMED AREA OF

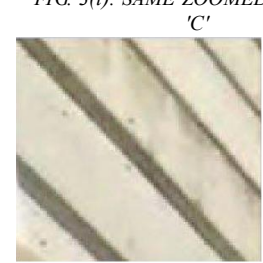

FIG. 3(k). SAME ZOOMED AREA OF 'E'

FIG. 3(l). SAME ZOOMED AREA OF ' $F$ '

FIG. 3. VISUAL COMPARISON OF 40 FRAME OF THE SEQUENCE "FOREMAN" WITH GAUSSIAN NOISE

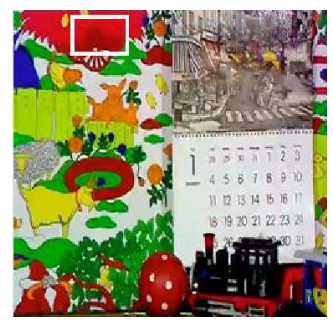

FIG. 4(a). ORIGINAL IMAGE

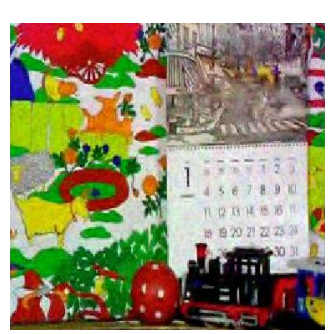

FIG. 4(e). CIFIC $(C P S N R=31.96 D B)$

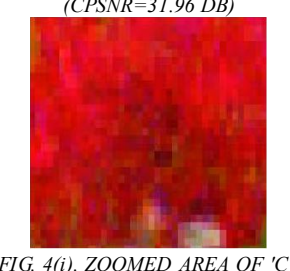

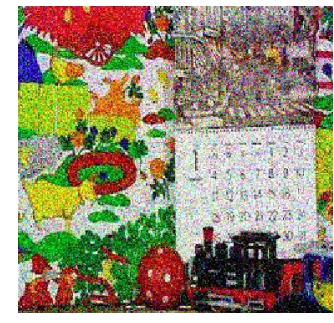

FIG. 4(b). NOISY IMAGE WITH

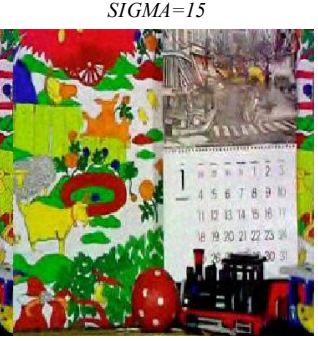

FIG. 4(f). PROPOSED METHOD

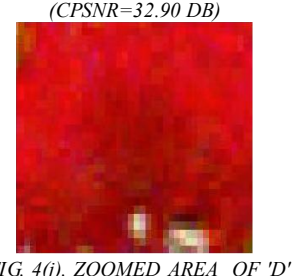

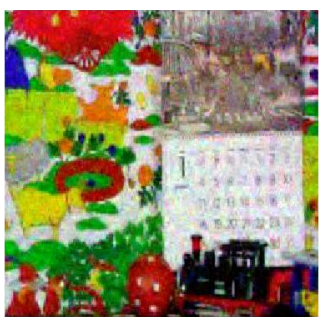

FIG. 4(c). NLM (CPSNR $=29.87 D B)$

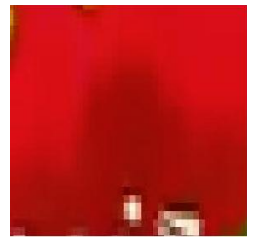

FIG. 4(g). ZOOMED AREA SHOWN

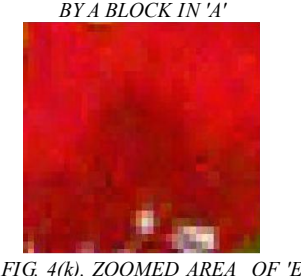

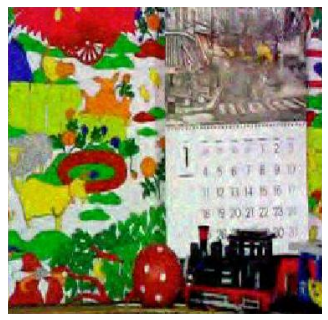

FIG. $4($ d). $V-B M 3 D$ $(C P S N R=30.95 D B)$

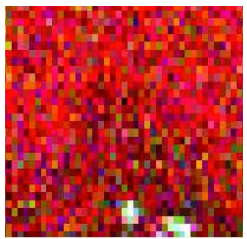

FIG. 4(h). ZOOMED AREA OF 'B'

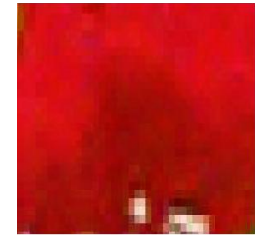

FIG. 4(l). ZOOMED AREA OF 'F'

FIG. 4. COMPARISON OF 35 FRAME OF "MOBILE" WITH UNIFORM GAUSSIAN NOISE AND ITS ZOOMED AREA SHOWN BY A BLOCK IN ORIGINAL FRAME

Mehran University Research Journal of Engineering \& Technology, Volume 38, No. 1, January, 2019 [p-ISSN: 0254-7821, e-ISSN: 2413-7219] 


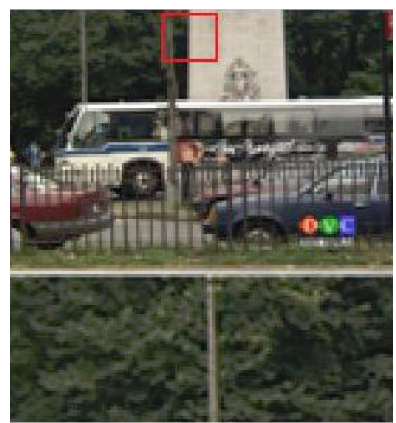

FIG. 5(a). ORIGINAL IMAGE

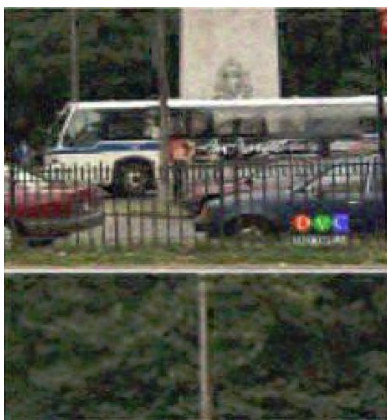

FIG. 5(e). CIFIC $(C P S N R=33.47 D B)$

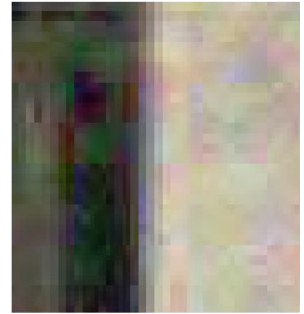

FIG. 5 (i) ZOOMED AREA OF 'C' (J)

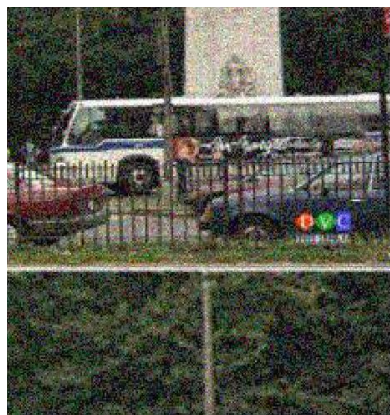

FIG. 5(b). NOISY IMAGE WITH SIGMA=15

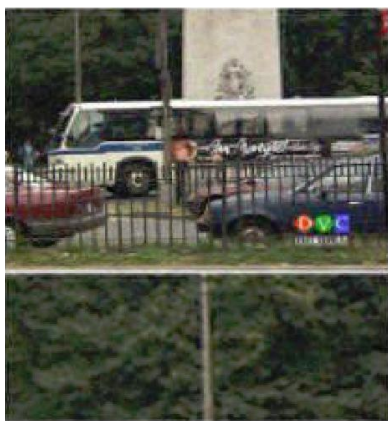

FIG. 5(f). PROPOSED METHOD $(C P S N R=34.85 \mathrm{DB})$

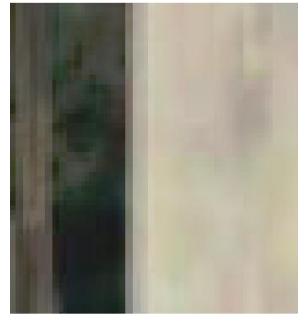

FIG. 5(j) ZOOMED AREA OF ' $C^{\prime}(J)$

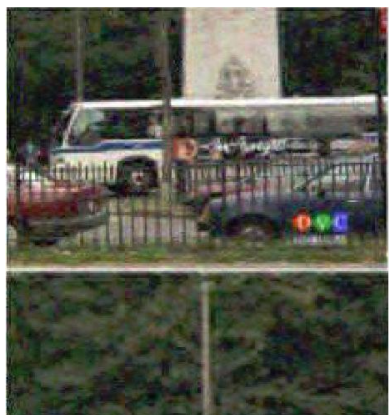

FIG. 5(c). $N L M(C P S N R=30.64 D B)$

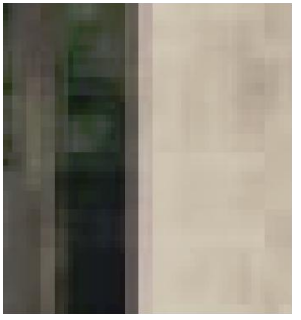

FIG. 5(g). ZOOMED AREA SHOWN BYA $B L O C K I N^{\prime} A$

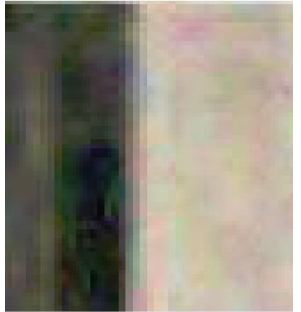

FIG. 5(k) ZOOMED AREA OF 'C' $(J)$

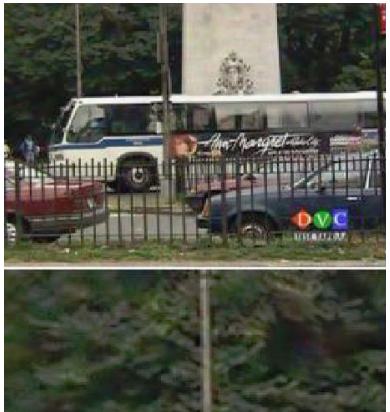

FIG. 5(d). V-BM3D (CPSNR=30.55 DB)

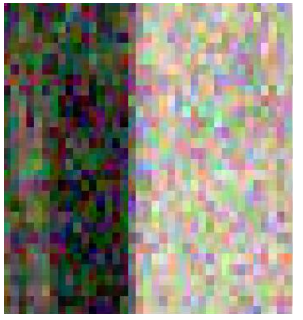

FIG 5(h). ZOOMED AREA OF 'B'

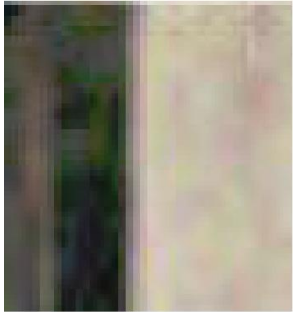

FIG. 5(l) ZOOMED AREA OF 'C' (J)

FIG. 5. COMPARISON OF 58 FRAME OF "BUS" WITH UNIFORM GAUSSIAN NOISE AND ITS ZOOMED AREA SHOWN BY A BLOCK IN ORIGINAL FRAME

Comparison of CPSNR for Different Sequences $(\sigma=15)$

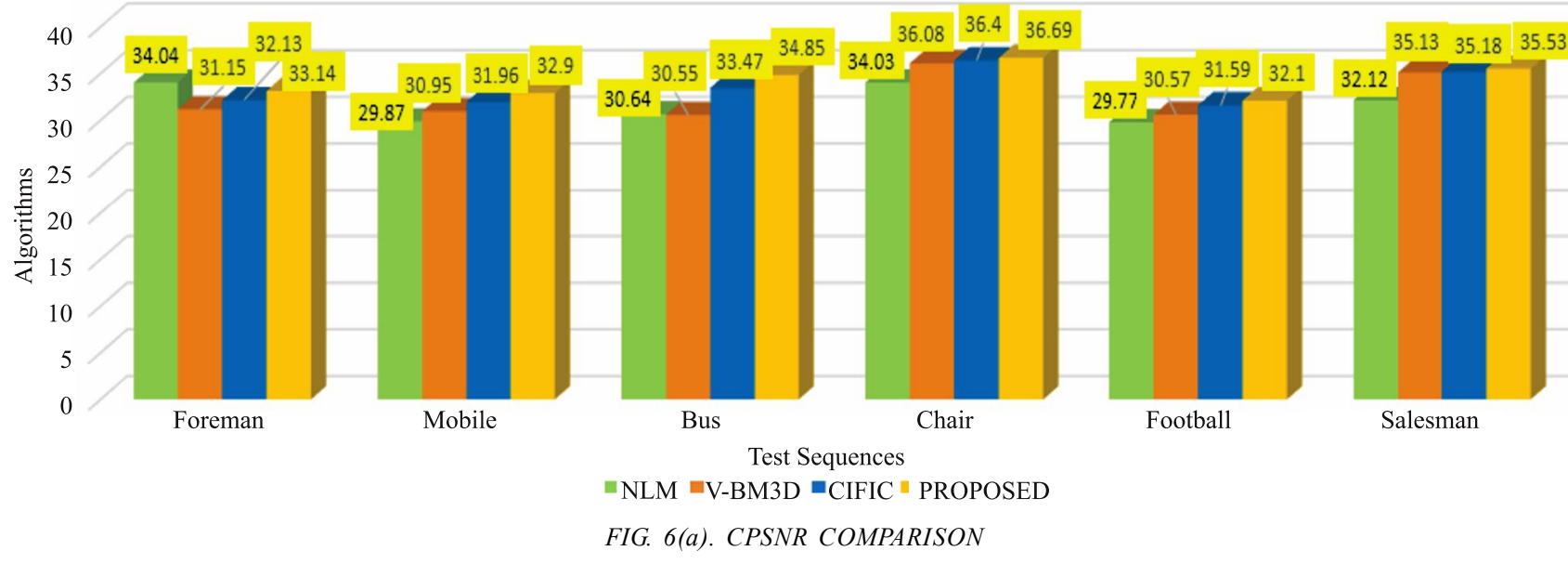

Mehran University Research Journal of Engineering \& Technology, Volume 38, No. 1, January, 2019 [p-ISSN: 0254-7821, e-ISSN: 2413-7219] 
Tables 3-4 presents a comparison of the proposed technique for speckle noise with existing state of-the-art algorithms. The comparison is shown in terms of PSNR (Peak Signalto-Noise Ratio), PCC, RMSE (Root Mean Square Error), and MSSIM.
The processing speed of NLMC and V-BM3D is higher about 10 seconds for one frame. On the other hand, the processing speed of the proposed technique is 5 seconds which is comparable to CIFIC. The proposed method is efficient to implement and has computational complexity comparable to existing state-of-the-art methods
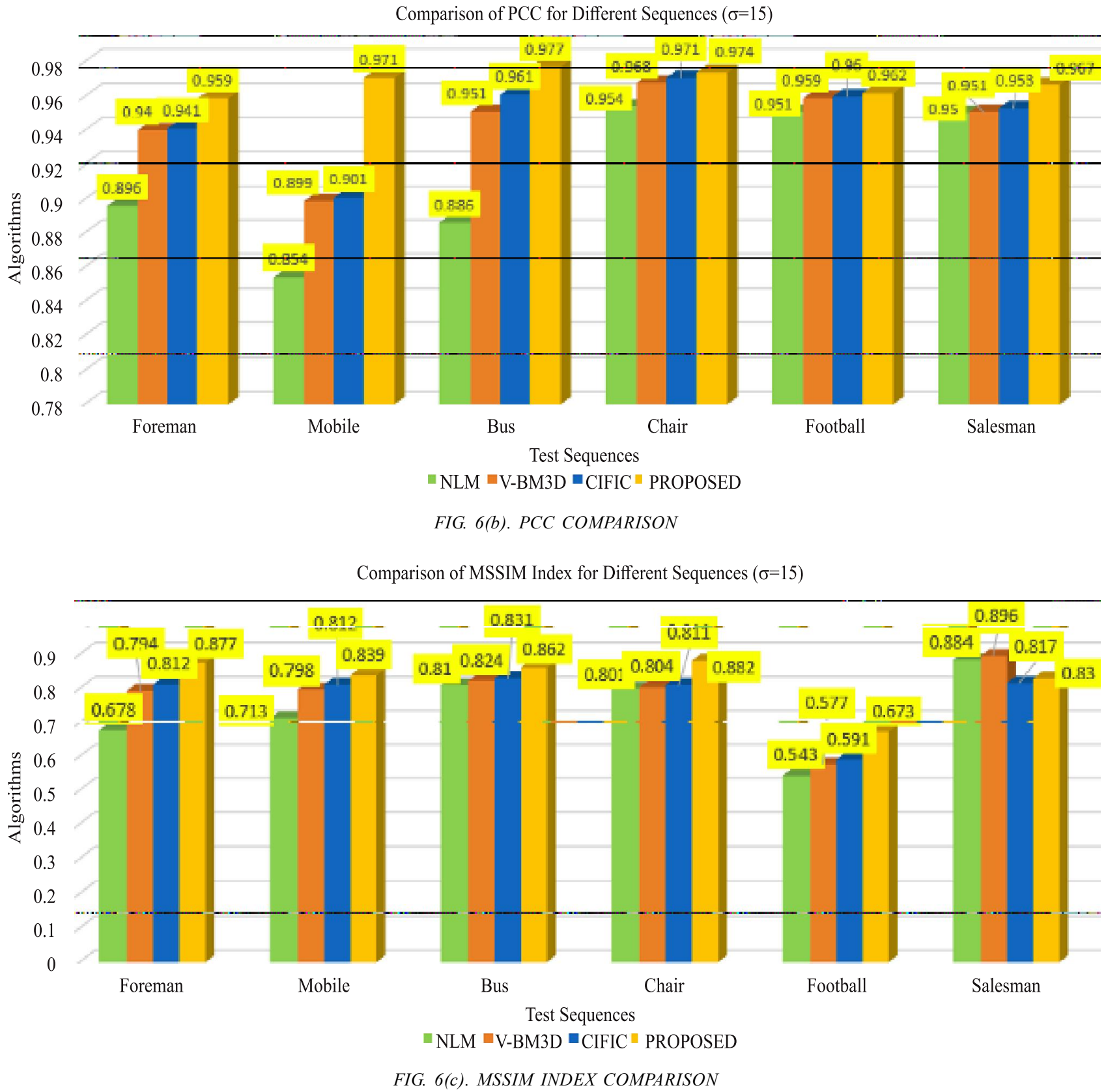

FIG. 6. GRAPHICAL COMPARISON OF DIFFERENT VIDEO SEQUENCES USING DIFFERENT TECHNIQUES 


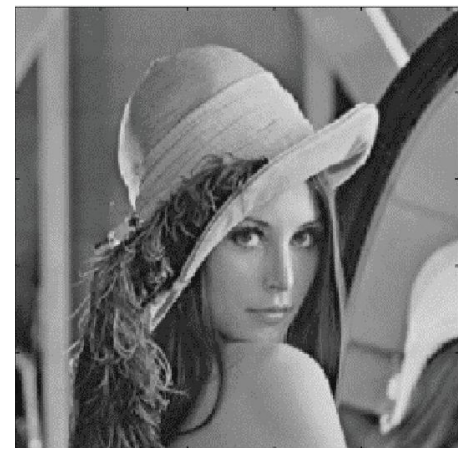

FIG. 7(a). ORIGINAL IMAGE

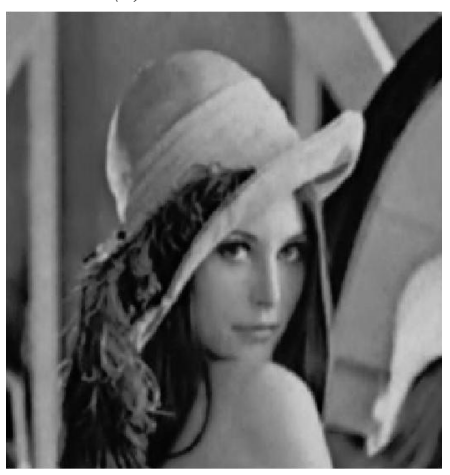

FIG.7(d). MD $(P S N R=26.51 \mathrm{DB})$

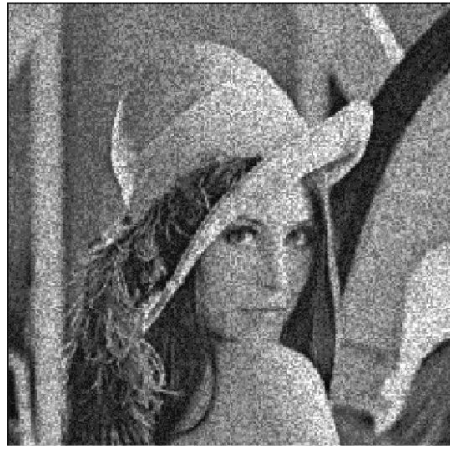

FIG. 7(b). NOISY IMAGE WITH SIGMA=0.04

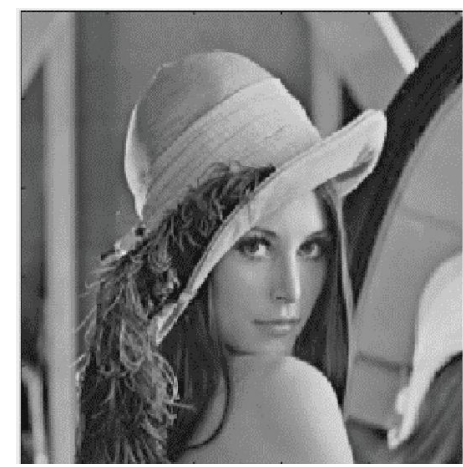

FIG. 7(e). WAVELETS $(P S N R=32.36 \mathrm{DB})$

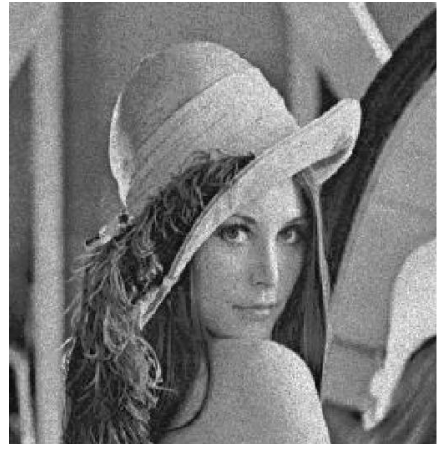

FIG. 7(c). SOMA $(P S N R=26.22 \mathrm{DB})$

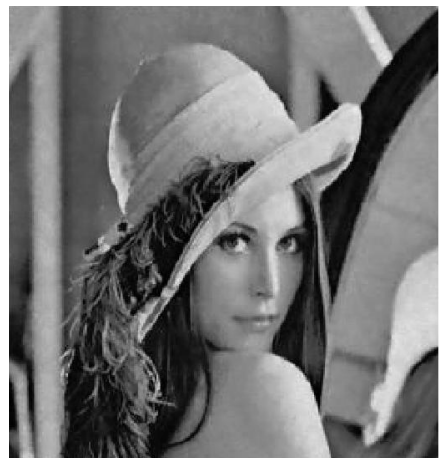

FIG.7(f). PROPOSED ALGORITHM $(P S N R=33.71 \mathrm{DB})$

FIG. 7. QUALITATIVE COMPARISON OF PROPOSED ALGORITHM WITH OTHER TECHNIQUES

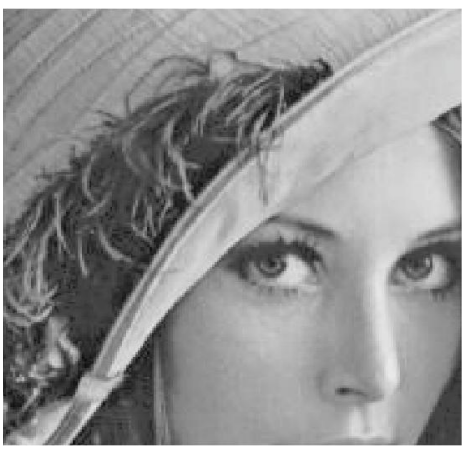

FIG. 8(a). ORIGINAL IMAGE

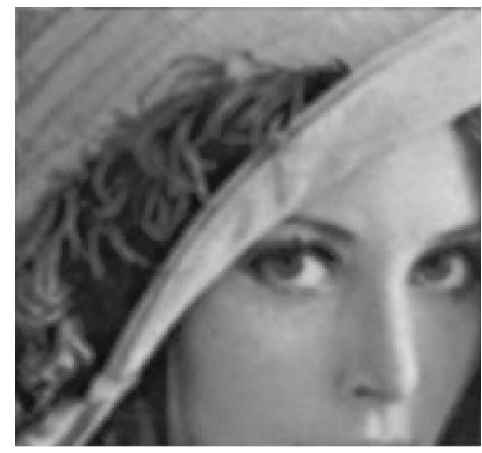

FIG. $8(d) . M D(P S N R=26.51 D B)$

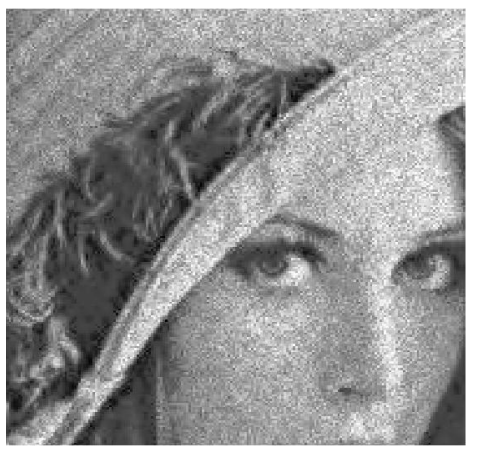

FIG. 8(b). NOISY IMAGE WITH SIGMA=0.04

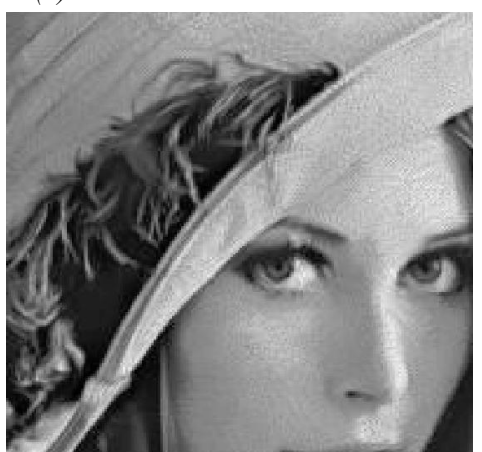

FIG. 8(e). WAVELETS $(P S N R=32.36 \mathrm{DB}$

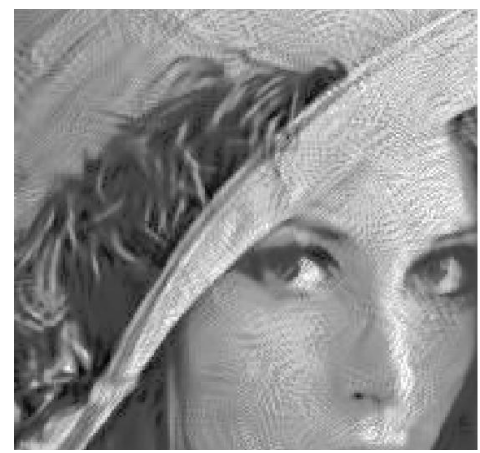

FIG. 8(c). SOMA (PSNR=26.22 DB)

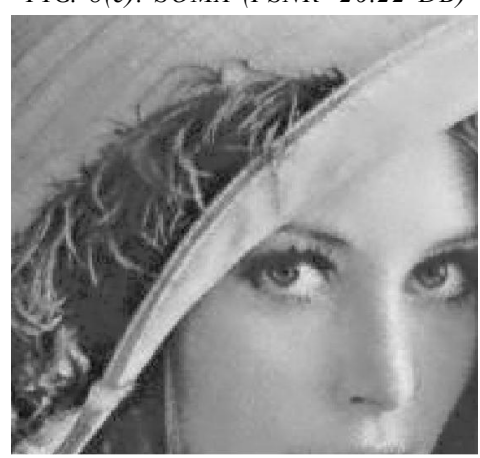

FIG. $8(f)$, PROPOSED ALGORITHM $(P S N R=33.71 \mathrm{DB})$

FIG. 8. QUALITATIVE COMPARISON OF ZOOMED LENA WITH OTHER TECHNIQUES 


\section{CONCLUSIONS}

This paper presents a video denoising technique that is based on the HMT model for color video denoising. It is used in the framework of 2D-GMM and 2D-DWT scales and location. DWT has a good performance in a task like a video compression and denoising. The excellence of this method is in parent-children correlation within and across the scales.
Experimental results reveal that the proposed method outperforms the existing state-of-the-art techniques for color video sequences both in terms of qualitative and quantitative analysis. This method is capable of noise reduction and edge preservation.

The computational complexity of the proposed algorithm is less in terms of its execution time as compared with NLMC, VBM3D and CIFIC. In future, this technique will be extended to high-resolution images.

Comparison of PSNR for Different Images

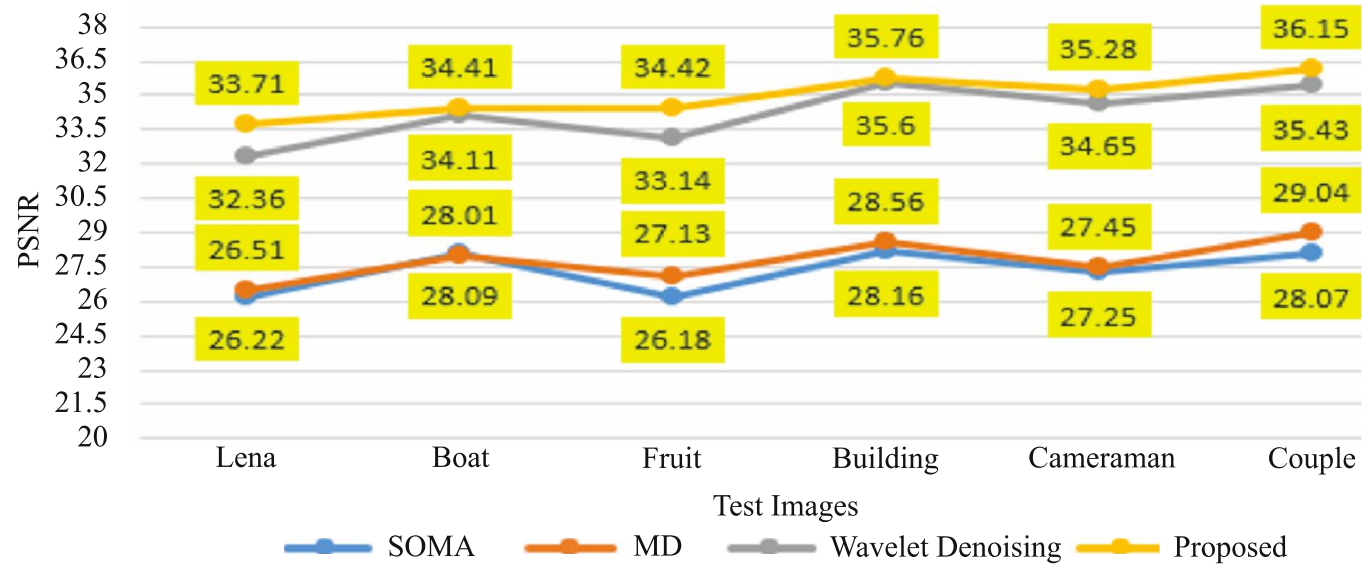

FIG. 9. GRAPHICAL COMPARISON OF PSNR OF DIFFERENT IMAGES USING DIFFERENT TECHNIQUES

TABLE 1. QUANTITATIVE COMPARISON OF NLM, V-BM3D, CIFIC AND PROPOSED WITH UNIFORM GAUSSIAN NOISE. ALL CPSNR VALUES ARE IN DECIBEL

\begin{tabular}{|c|c|c|c|c|c|c|c|}
\hline \multirow{3}{*}{ Algorithm } & \multirow{3}{*}{$\begin{array}{l}\text { Quantitative } \\
\text { Measure }\end{array}$} & \multicolumn{6}{|c|}{$\sigma_{n}=15$} \\
\hline & & \multicolumn{6}{|c|}{ Video Sequences } \\
\hline & & Foreman & Mobile & Bus & Chair & Football & Salesman \\
\hline \multirow{3}{*}{ NLM } & CPSNR & 34.04 & 29.87 & 30.64 & 34.03 & 29.77 & 32.12 \\
\hline & PCC & 0.896 & 0.854 & 0.886 & 0.954 & 0.951 & 0.950 \\
\hline & MSSIM Index & 0.678 & 0.713 & 0.810 & 0.801 & 0.543 & 0.884 \\
\hline \multirow{3}{*}{ V-BM3D } & CPSNR & 31.15 & 30.95 & 30.55 & 36.08 & 30.57 & 35.13 \\
\hline & PCC & 0.940 & 0.899 & 0.951 & 0.968 & 0.959 & 0.951 \\
\hline & MSSIM Index & 0.794 & 0.798 & 0.824 & 0.804 & 0.577 & 0.896 \\
\hline \multirow{3}{*}{ CIFIC } & CPSNR & 32.13 & 31.96 & 33.47 & 36.40 & 31.59 & 35.18 \\
\hline & PCC & 0.941 & 0.901 & 0.961 & 0.971 & 0.960 & 0.953 \\
\hline & MSSIM Index & 0.812 & 0.812 & 0.831 & 0.811 & 0.591 & 0.817 \\
\hline \multirow{3}{*}{$\begin{array}{l}\text { Proposed } \\
\text { Algorithm }\end{array}$} & CPSNR & 33.14 & 32.90 & 34.85 & 36.69 & 32.10 & 35.53 \\
\hline & PCC & 0.959 & 0.971 & 0.977 & 0.974 & 0.962 & 0.967 \\
\hline & MSSIM Index & 0.877 & 0.839 & 0.862 & 0.882 & 0.673 & 0.830 \\
\hline
\end{tabular}

Mehran University Research Journal of Engineering \& Technology, Volume 38, No. 1, January, 2019 [p-ISSN: 0254-7821, e-ISSN: 2413-7219] 
TABLE 2. QUANTITATIVE COMPARISON OF NLM, V-BM3D, CIFIC AND PROPOSED WITH UNIFORM GAUSSIAN NOISE. ALL CPSNR VALUES ARE IN DECIBEL

\begin{tabular}{|c|c|c|c|c|c|c|c|}
\hline \multirow{3}{*}{ Algorithm } & \multirow{3}{*}{$\begin{array}{l}\text { Quantitative } \\
\text { Measure }\end{array}$} & \multicolumn{6}{|c|}{$\sigma_{n}=15$} \\
\hline & & \multicolumn{6}{|c|}{ Video Sequences } \\
\hline & & Foreman & Mobile & Bus & Chair & Football & Salesman \\
\hline \multirow{3}{*}{ NLM } & CPSNR & 33.09 & 28.17 & 27.82 & 31.07 & 27.32 & 29.03 \\
\hline & PCC & 0.765 & 0.851 & 0.876 & 0.945 & 0.922 & 0.914 \\
\hline & MSSIM Index & 0.564 & 0.710 & 0.798 & 0.796 & 0.458 & 0.751 \\
\hline \multirow{3}{*}{ V-BM3D } & CPSNR & 30.11 & 29.15 & 27.81 & 33.91 & 27.80 & 32.13 \\
\hline & PCC & 0.870 & 0.814 & 0.893 & 0.911 & 0.943 & 0.877 \\
\hline & MSSIM Index & 0.685 & 0.688 & 0.744 & 0.789 & 0.389 & 0.810 \\
\hline \multirow{3}{*}{ CIFIC } & CPSNR & 30.16 & 30.06 & 30.51 & 33.71 & 28.81 & 32.10 \\
\hline & PCC & 0.876 & 0.866 & 0.899 & 0.854 & 0.912 & 0.945 \\
\hline & MSSIM Index & 0.723 & 0.765 & 0.743 & 0.712 & 0.412 & 0.789 \\
\hline \multirow{3}{*}{$\begin{array}{l}\text { Proposed } \\
\text { Algorithm }\end{array}$} & CPSNR & 32.14 & 31.60 & 31.13 & 33.89 & 29.35 & 33.23 \\
\hline & PCC & 0.893 & 0.912 & 0.915 & 0.945 & 0.933 & 0.926 \\
\hline & MSSIM Index & 0.743 & 0.814 & 0.827 & 0.867 & 0.594 & 0.819 \\
\hline
\end{tabular}

TABLE 3. QUANTITATIVE RESULTS OF PROPOSED ALGORITHM WITH OTHER TECHNIQUES WITH SPECKLE NOISE AND UNIFORM NOISE LEVEL

\begin{tabular}{|c|c|c|c|c|c|c|c|}
\hline \multirow{2}{*}{ Algorithm } & \multirow{2}{*}{$\begin{array}{l}\text { Quantitative } \\
\text { Measure }\end{array}$} & \multicolumn{6}{|c|}{ Test Images } \\
\hline & & Foreman & Mobile & Bus & Chair & Football & Salesman \\
\hline \multirow{4}{*}{ SOMA } & PSNR & 26.22 & 28.09 & 26.18 & 28.16 & 27.25 & 28.07 \\
\hline & RMSE & 137.02 & 132.028 & 130.714 & 133.93 & 135.47 & 131.35 \\
\hline & PCC & 0.9521 & 0.9723 & 0.8725 & 0.9821 & 0.9617 & 0.8983 \\
\hline & MSSIM Index & 0.7163 & 0.7805 & 0.7678 & 0.7782 & 0.7852 & 0.9609 \\
\hline \multirow{4}{*}{ MD } & PSNR & 26.51 & 28.01 & 27.13 & 28.56 & 27.45 & 29.04 \\
\hline & RMSE & 132.22 & 129.98 & 130.71 & 129.13 & 126.18 & 131.35 \\
\hline & PCC & 0.9573 & 0.9745 & 0.9617 & 0.9894 & 0.9667 & 0.9941 \\
\hline & MSSIM Index & 0.7181 & 0.7962 & 0.7813 & 0.7890 & 0.7884 & 0.9621 \\
\hline \multirow{4}{*}{$\begin{array}{l}\text { Wavelet } \\
\text { Denoising }\end{array}$} & PSNR & 32.36 & 34.11 & 33.14 & 35.60 & 34.65 & 35.43 \\
\hline & RMSE & 98.871 & 96.219 & 94.337 & 95.144 & 125.819 & 98.761 \\
\hline & PCC & 0.9924 & 0.9933 & 0.9925 & 0.9889 & 0.9954 & 0.9981 \\
\hline & MSSIM Index & 0.9233 & 0.8785 & 0.8962 & 0.9045 & 0.8495 & 0.9233 \\
\hline \multirow{4}{*}{$\begin{array}{l}\text { Proposed } \\
\text { Algorithm }\end{array}$} & PSNR & 33.71 & 34.41 & 34.42 & 35.76 & 35.28 & 36.15 \\
\hline & RMSE & 108.671 & 99.932 & 94.376 & 105.141 & 128.659 & 138.651 \\
\hline & PCC & 0.9983 & 0.9964 & 0.9948 & 0.9991 & 0.9974 & 0.9987 \\
\hline & MSSIM Index & 0.9353 & 0.8789 & 0.9162 & 0.9154 & 0.8953 & 0.9451 \\
\hline
\end{tabular}

Mehran University Research Journal of Engineering \& Technology, Volume 38, No. 1, January, 2019 [p-ISSN: 0254-7821, e-ISSN: 2413-7219] 


\begin{tabular}{|c|c|c|c|c|c|c|}
\hline \multirow[b]{2}{*}{$\begin{array}{c}\text { Quantitative } \\
\text { Measures }\end{array}$} & \multirow[b]{2}{*}{ Image Set } & \multicolumn{5}{|c|}{ Speckle } \\
\hline & & $\begin{array}{l}\text { Frame } 1=0.03 \\
\text { Frame } 2=0.03 \\
\text { Frame } 3=0.03\end{array}$ & $\begin{array}{l}\text { Frame } 1=0.02 \\
\text { Frame } 2=0.04 \\
\text { Frame } 3=0.06\end{array}$ & $\begin{aligned} \text { Frame } 1 & =0.06 \\
\text { Frame } 2 & =0.06 \\
\text { Frame } 3 & =0.06\end{aligned}$ & $\begin{array}{l}\text { Frame } 1=0.06 \\
\text { Frame } 2=0.08 \\
\text { Frame } 3=0.10\end{array}$ & $\begin{array}{l}\text { Frame } 1=0.09 \\
\text { Frame } 2=0.09 \\
\text { Frame } 3=0.09\end{array}$ \\
\hline \multirow{5}{*}{ PSNR } & Lena & 33.714 & 32.412 & 31.644 & 31.174 & 30.135 \\
\hline & Boat & 34.416 & 34.112 & 32.865 & 31.461 & 31.179 \\
\hline & Fruit & 34.423 & 33.145 & 32.617 & 32.071 & 30.561 \\
\hline & Building & 35.763 & 34.441 & 33.091 & 32.982 & 30.143 \\
\hline & Cameraman & 35.286 & 33.982 & 32.981 & 32.132 & 30.013 \\
\hline \multirow{5}{*}{ RMSE } & Lena & 108.671 & 110.081 & 116.284 & 116.887 & 118.093 \\
\hline & Boat & 99.932 & 103.012 & 104.016 & 105.018 & 105.091 \\
\hline & Fruit & 94.376 & 95.714 & 95.976 & 96.019 & 97.023 \\
\hline & Building & 105.141 & 106.109 & 108.512 & 108.854 & 109.158 \\
\hline & Cameraman & 128.659 & 129.008 & 129.841 & 130.153 & 130.816 \\
\hline \multirow{5}{*}{ PCC } & Lena & 0.9983 & 0.9895 & 0.9851 & 0.9822 & 0.9813 \\
\hline & Boat & 0.9964 & 0.9951 & 0.9948 & 0.9941 & 0.9916 \\
\hline & Fruit & 0.9948 & 0.9940 & 0.9936 & 0.9927 & 0.9918 \\
\hline & Building & 0.9891 & 0.9861 & 0.9823 & 0.9819 & 0.9811 \\
\hline & Cameraman & 0.9974 & 0.9952 & 0.9923 & 0.9896 & 0.9881 \\
\hline \multirow{5}{*}{$\begin{array}{l}\text { MSSIM } \\
\text { Index }\end{array}$} & Lena & 0.9353 & 0.9351 & 0.9344 & 0.9342 & 0.9339 \\
\hline & Boat & 0.8789 & 0.8775 & 0.8764 & 0.8732 & 0.8713 \\
\hline & Fruit & 0.9162 & 0.9158 & 0.9151 & 0.9149 & 0.9143 \\
\hline & Building & 0.9154 & 0.9147 & 0.9141 & 0.9138 & 0.9131 \\
\hline & Cameraman & 0.8953 & 0.8951 & 0.8946 & 0.8941 & 0.8940 \\
\hline
\end{tabular}

\section{ACKNOWLEDGEMENT}

Authors take this opportunity to extend their profound gratitude to Military College of Signals, National University of Sciences \& Technology, Rawalpindi, Pakistan, for research funding and anonymous reviewers/experts for their valuable comments and feedback.

\section{REFERENCES}

[1] Sharma, A., and Singh, J., "Image Denoising Using Spatial Domain Filters: A Quantitative Study", Proceedings of $6^{\text {th }}$ IEEE International Congress on Image and Signal Processing, Hangzhou, China, December, 2013.
[2] Narasimha, C., and Rao, N.A., "Spatial Domain Filter for Medical Image Enhancement”, Proceedings of IEEE International Conference on Signal Processing and Communication Engineering Systems,Guntur, India, January, 2015.

[3] Wang, B., Xiong, Z., and Zhang, D., "Nonlocal Image Denoising via Collaborative Spatial-domain LMMSE Estimation", IEEE International Conference on Image Processing, Paris, France, October, 2014.

[4] Lee, J.S., "Digital Image Enhancement and Noise Filtering by Use of Local Statistics", IEEE Transactions on Pattern Analysis and Machine Intelligence, Volume 2, No. 2, pp.165-168, 1980 . 
[5] Li, X., Shen, H., and Zhang, L., "Sparse-Based Reconstruction of Missing Information in Remote Sensing Images from Spectral/Temporal Complementary Information", ISPRS Journal of Photogrammetry and Remote Sensing, Volume 106, pp. 1-15, 2015.

Ozkan, M.K., Sezan, M.I., and Tekalp, A.M., "Adaptive Motion-Compensated Filtering of Noisy Image Sequences", IEEE Transactions on Circuits and Systems for Video Technology, Volume3, No.4, pp. 277-290, 1993.

[7] Liu, Y., and Luo, B., "Weighting Wiener and Total Variation for Image Denoising", Proceedings of IEEE International Conference on Information and Automation Ningbo, China, August, 2016.

Maggioni, M., Monge, E.S., and Foi, A., "Joint Removal of Random and Fixed-Pattern Noise through Spatiotemporal Video Filtering", IEEE Transactions on Image Processing, Volume23, No. 10, pp.4282-4296, 2014.

Wang, X., Zhu, C., and Li, S., "Depth Filter Design by Jointly Utilizing Spatial-Temporal Depth and Texture Information", IEEE International Symposium on Broadband Multimedia Systems and Broadcasting, pp. 1-5, Ghent, Belgium, June, 2015.

[10] Crouse, M.S., Nowak, R.D., and Baraniuk, R.G., "WaveletBased Statistical Signal Processing using Hidden Markov Models", IEEE Transactions on Signal Processing, Volume 46, No. 4, pp. 886-902, 1998.

[11] Malfait, M., and Roose, D., "Wavelet-Based Image Denoising Using a Markov Random Field a Prior Model", IEEE Transactions on Image Processing, Volume 6, No. 4, pp. 549-565, 1997.

[12] Wen, B., Ravishankar, S., and Bresler, Y., "Video Denoising by Online 3D-Sparsifying Transform Learning”, IEEE International Conference on Image Processing, pp. 1-5, Quebec City, Canada, September 2015.

[13] Zhigang, D., Jingxuan, Z., and Chunrong J., "An Improved Wavelet Threshold Denoising Algorithm", Intelligent System Design and Engineering Applications, Hong Kong, China, January, 2013.

[14] Riaz, M.U., Touqir, I., and Haider, M., "Wavelet-Based Image Modelling for Compression Using Hidden Markov Model", International Journal of Advanced Computer Science and Applications, Volume 7, No. 8, pp. 1-7, 2016.
[15] Ho, J., and Hwang, W.L., "Wavelet Bayesian Network Image Denoising", IEEE Transactions on Image Processing, Volume 22, No. 4, 2014.

[16] Dabov, K., Foi, A., and Egiazarian, K., "Video Denoising by Sparse 3D-Transform-Domain Collaborative Filtering", Proceedings of 15 th European Signal Processing Conference, Poznan, Poland, 2007.

[17] Maggioni, M., Katkovnik, V., and Egiazarian, K., "Nonlocal Transform-Domain Filter for Volumetric Data Denoising and Reconstruction", IEEE Transactions on Image Processing, Volume 22, No. 1, pp. 119-133, January, 2013.

[18] Zuo, C., Liu, Y., and Tan, X., "Video Denoising Based on a Spatiotemporal Kalman-Bilateral Mixture Model", The Scientific World Journal, pp. 1-10, 2013.

[19] Aydin, V.A., and Foroosh, H., "Motion-Compensated Temporal Filtering for Critically-Sampled WaveletEncoded Images",Computer Vision and Pattern Recognition, Cornell University Library, May, 2017.

[20] Hong-Zhi, W., Ling, C., and Shu-Liang, S., "Improved Video Denoising Algorithm Based on Spatial-Temporal Combination", Proceedings of IEEE 7th International Conference on Image and Graphics, pp. 64-67, Qingdao, China, July, 2013.

[21] Donoho, D.L., and Johnstone, I.M., "Ideal Spatial Adaptation via Wavelet Shrinkage", Biometrika, Volume 81, pp. 425-455, 1994.

[22] Haider, M., Touqir, I., Riaz, M.U., Siddiqui, A.M.,"Denoising in Wavelet Domain Using Probabilistic Graphical Models", International Journal of Advanced Computer Science and Applications, Volume 7, No. 11, 2016 .

[23] Dai, J., C. Au, O., Pang, C., and Zou, F., "Colour Video Denoising Based on Combined Interframe and Intercolor Prediction", IEEE Transactions on Circuits and Systems for Video Technology, Volume 23, No. 1, 2013.

[24] Anupriya, A., and Tayal, A., "Wavelet Based Image Denoising using Self Organizing Migration Algorithm", CIIT International Journal of Digital Image Processing, Volume 4, No.10, pp. 542-546, 2012.

[25] Knaus, C., and Zwicker, N., "Dual-Domain Image Denoising", Proceedings of IEEE International Conference on Image Processing, pp. 440-444, Melbourne, Australia, September, 2013.

[26] Habib, W., Sarwar, T., and Masood, A., "Wavelet Denoising of Multi-Frame Optical Coherence Tomography Data Using Similarity Measures", IET Image Processing, Volume 11, No. 1, pp. 64-79, 2017. 